\title{
Surgical Management by Standing Laparoscopy and Inguinal Celiotomy Approach of the Cryptorchid Canadian Pony Stallion - Case Report
}

\author{
Cornel IGNA ${ }^{1 *}$, Roxana DASCALU ${ }^{1}$, Daniel BUMB ${ }^{1}$, Bogdan SICOE ${ }^{1}$, Larisa SCHUSZLER ${ }^{1}$ \\ ${ }^{1}$ Banat's University of Agricultural Science and Veterinary Medicine, "King Michael I of Romania" from \\ Timisoara, Romania \\ *corresponding author: ignacornel@gmail.com
}

Bulletin UASVM Veterinary Medicine 73(2) / 2016,

Print ISSN 1843-5270; Electronic ISSN 1843-5378

DOI:10.15835/buasvmcn-vm: 12071

\begin{abstract}
Laparoscopic surgery has become an accepted method of identification and removal of intra-abdominal testes in the horse. In the veterinary literature there are few reports in which standing laparoscopic cryptorchidectomy could not be performed in stallions, including miniature horse too. This report describes the surgical management of a Canadian pony diagnosed with partial abdominal unilaterally cryptorchidism at which laparoscopic castration has failed and the removal of the retained testicle has been made through inguinal celiotomy. A 4-year-old unilaterally cryptorchid Canadian pony was admitted in the Surgery Clinic of the Faculty of Veterinary Medicine Timișoara for castration. The left testis was in the scrotum, but neither the right testis nor the right epididymis could be located by external palpation of the right inguinal canal. The proposed treatment was laparoscopic cryptorchidectomy with pony standing in stock. With a long-handled forceps inserted into the abdominal cavity, the testis cord was gently grasped and moderate traction was applied without success in bringing the testicle into the abdomen (retained testis). After the failure of laparascopic cryptorchidectomy but having an exact diagnosis (partial abdominal unilaterally cryptorchidism), we have proceeded to open cryptorchidectomy via inguinal approach with pony anesthetized and positioned in dorsal recumbency. This case report reveals that the major disadvantage of the cryptorchidectomy by flank approach is the inability to remove an inguinally retained testis.

Conclusion: If the location of the testis is unknown, the standing laparoscopic diagnose can be decisive. Cryptorchidectomy via inguinal approach and noninvasive method for identifying and everting the vaginal process by traction of the inguinal extension of the gubernaculum testis are commonly facile procedure, and this report describes, according to our knowledge, that this procedure has been performed for the first time in Romania.
\end{abstract}

Keywords: cryptorchidectomy, inguinal celiotomy, laparoscopic, stallion pony

\section{INTRODUCTION}

Cryptorchidism is a term used to describe the congenital condition in which one or both testicles fail to descend into the scrotum (Shira and Genetzky, 1982). The causes of cryptorchidism are not known, but testicular abnormalities have been suggested (Gardner et al., 2016).

Cryptorchid stallions often display the physical and behavioral characteristics of normal stallions. To eliminate masculine characteristics, both testes are routinely entirely removed at cryptorchid stallions (McKinnon et al., 2011; Rodgerson and Hanson, 1997).

The numerous approaches for cryptorchidectomy that have been used in horses include: inguinal (Adams and Fessler, 2000; Valdez et al., 1979; Walker and Vaughan, 1980), parainguinal (McKinnon et al., 2011), modified parainguinal (Wilson and Reinertson, 1987), suprapubic paramedian (Cox et al., 1978) and paralumbar flank (Swift, 1972). Each procedure has advantages and disadvantages (Adams and Fessler, 2000; 
McKinnon et al., 2011; Rodgerson and Hanson, 1997).

Abdominal testes also can be removed by laparoscopic techniques (Davis, 1997; Fischer and Vachon, 1992; Fischer, 1991; Hanrath and Rodgerson, 2002; Hendrickson and Wilson, 1997; Hendrickson, 2008; Ragle et al., 1998; Wilson et al., 1996; Wilson and Madison, 1989).

Laparoscopic surgery has become an accepted method of identification and removal of intra-abdominal testes in the horse. Wilson and Madison (1989) describe the use of laparoscopy to diagnose abdominal retained testes. Fischer (1991) was the first who describe a laparoscopic cryptorchidectomy technique. Since then, numerous laparoscopic cryptorchidectomy techniques have been described, which vary in the positioning of the horse (standing versus dorsal recumbency), anesthetic techniques (Muir et al., 2000 ), position and number of the portals, and the method used to provide hemostasis of the spermatic cord (Hendrickson, 2012).

The mono or bipolar electrosurgical devices were successfully used in cryptorchidectomy (Hanrath and Rodgerson, 2002; Hendrickson, 2006; Wilson et al., 1996). Fischer and Vachon (1992) says that the most frequently employed and most cost effective method for laparoscopic intra-abdominal removal of cryptorchid testes is the combined use of an endoscopic ligating loop and monopolar electrosurgery.

So far, in the encountered specialty literature there are few reports in which standing laparoscopic cryptorchidectomy could not be performed in stallions including miniature horse too (McKinnon et al., 2011).

This report describes the surgical management of a Canadian pony diagnosed with partial abdominal unilaterally cryptorchidism at which laparoscopic castration has been failed and the removal of retained testicle has been made through inguinal celiotomy.

\section{MATERIALS AND METHODS}

A 4-year-old unilaterally cryptorchid Canadian pony was admitted to the Surgery Clinic of Banat's University of Agricultural Sciences and Veterinary Medicine - Timisoara for castration. The left testis was in the scrotum, but neither the right testis nor the right epididymis could be located by external palpation of the right inguinal canal.
Rectal palpation of the testis and right vaginal ring was not attempted, because the patient was a miniature pony.

The diagnosis stated was complete or partial abdominal unilaterally cryptorchidism according with van der Velden study (1990). The proposed treatment was laparoscopic cryptorchidectomy with pony standing in stock (Davis, 1997; Fischer and Vachon, 1992; Fischer, 1991; Rodgerson and Hanson, 1997).

Preoperatory food was withheld for 24 hours to reduce the amount of intestinal contents. This increases surgical visibility and maneuverability, as well as reduces the risk of inadvertent puncture of viscera (Rodgerson and Hanson, 1997). With pony sedated (detomidine - $0.03 \mathrm{mg} / \mathrm{kg}$ and butorphanol $0.04 \mathrm{mg} / \mathrm{kg}$; direct infiltration of the paralumbar fossa with lidocaine) and restrained in stocks, the tail is tied and the paralumbar fossa was prepared for surgery in routine fashion and draped.

The trocar-cannula (Karl Storz - 11mm), was inserted through the right paralumbar fossa and the abdominal cavity was insufflated with $\mathrm{CO}_{2}$. The trocar (upon cannula with multifunctional valve) was replaced by the laparoscope (Karl Storz Hopkins, oblique $30^{\circ} / 10 \mathrm{~mm} / 31 \mathrm{~cm}$ ) and the caudal portion of the abdomen was examined to identify the retained testis. The vaginal ring was located and the right spermatic cord was identified to penetrate the right profound inguinal ring. Since the deep palpation of the external inguinal ring was not managed by bringing the testicle in the abdomen, a second incision in the flank was performed, $10 \mathrm{~cm}$ caudoventral to the laparoscope portal, and the instrument portal was engaged into the abdomen (Figure 1). With a long-handled forceps (Karl Storz - Clickline Babcock grasping forceps $10 \mathrm{~mm} / 43 \mathrm{~cm}$ ) inserted into the abdominal cavity, the testis cord was gently grasped and moderate traction was applied, without success in bringing the testicle into the abdomen (retained testis) (Figure 2).

After the failure of laparascopic cryptorchidectomy, but having an exact diagnosis - partial abdominal right unilaterally cryptorchidism, we have proceeded to open cryptorchidectomy via inguinal approach with pony anesthetized and positioned in dorsal recumbency.

The abdomen was deflated through a laparoscopic cannula. Closure of laparoscopic portals 


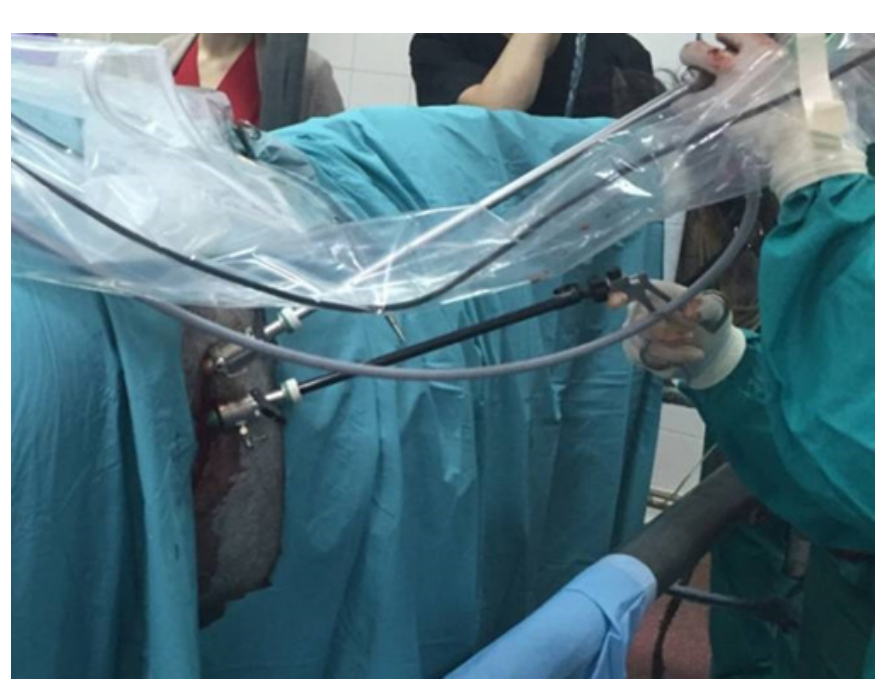

Fig. 1. - Laparoscopy

was performed in one layer, using monofilament suture materials (Coated Vicryl, Polyglactin 910, 3 Ph. Eur., Ethicon).

Initial anesthesia was supplemented with guaifenesin administered IV for induction, endotracheal intubation was then performed and followed by maintenance with 3\% isoflurane in oxygen, in semi-closed respiratory circular system with spontaneous breathing.

An elliptical incision for bursectomy (scrotal excision) was made (Palmer and Passmore, 1987), and the right superficial inguinal ring and left testis have been identified (Figure 3a).

The right testis was retained in the inguinal canal. The gubernaculum testis was located by careful examination of the superficial inguinal ring's margin (Rodgerson and Hanson, 1997; Searle et al., 1999) (Figure 3a). By gently grasping and retraction of gubernaculum testis (inguinal extension) the vaginal process that was inverted in the inguinal canal was everted into canal, where was exteriorized (Figure $3 \mathrm{~b}$ ). The vaginal sac was identified (Figure 3c) and opened. The proper ligament of the testis was identified, extraabdominal retracted and classically removed (Figure 3d). The vaginal process, the superficial inguinal ring, and the subcutaneous tissue were closed separately with continuous simple suture (PDS II, Polydioxanone, 2 Ph. Eur., Ethicon). The skin was closed with interrupted sutures. The antibiotics (large broad spectrum, Depocillin, Intervet) and tetanus (Ser Clostetan, Bioveta) prophylaxis were performed postoperatively.

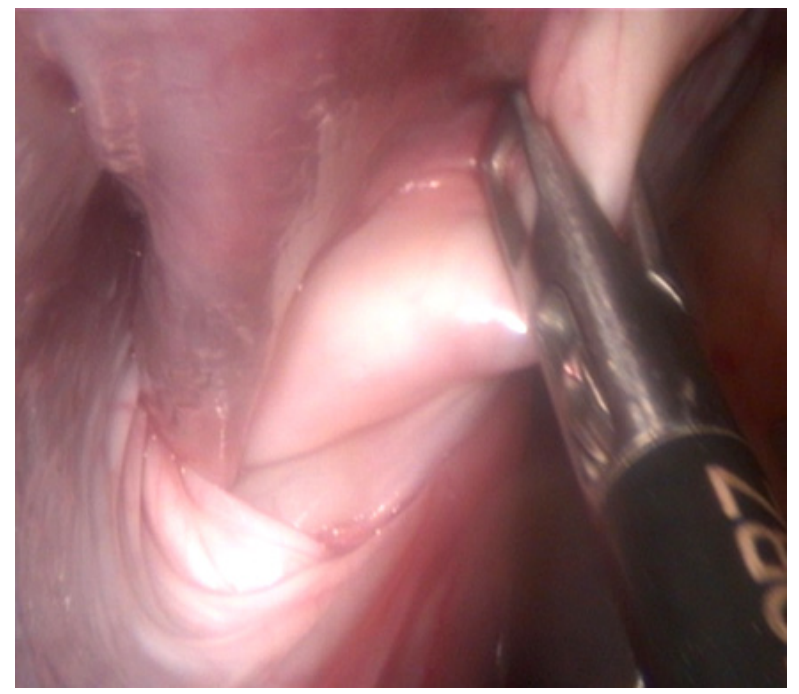

Fig. 2 - The right vaginal ring and spermatic cord

Postoperative diagnosis was revised so partial abdominal unilaterally (right) cryptorchidism with retained testis in the inguinal canal was observed. Cryptorchidism was probably caused by testicular hypoplasia after anterior trauma (Figure 4).

\section{RESULTS AND DISCUSSION}

This case report reveals that the major disadvantage of the cryptorchidectomy by flank approach is the inability to remove an inguinally retained testis, conclusion also mentioned in another study (Rodgerson and Hanson, 1997). In a study performed on ten horses with unilaterally or bilaterally retained testes, the authors reached to the conclusion that standing laparoscopic cryptorchidectomy can be performed easily and safely using electrosurgical instrumentation as the sole means of providing hemostasis of the equine mesorchium. (Hanrath and Rodgerson, 2002).

The abandonment of the laparoscopic removal of the cryptorchid testis and the use of open cryptorchidectomy by inguinal approach is explainable, on the one hand by retention of the right testis in the inguinal canal, and on the other hand by arguments of Voermans and al. study (Voermans et al., 2006). These authors consider, after intra-abdominal transection of the spermatic cord, $5.6 \%$ of inguinally retained and $3.4 \%$ of normally descended testes failed to become completely necrotic, as a result of an alternate blood supply via the cremasteric and/or external pudendal artery. Therefore, laparoscopic castration without orchidectomy 


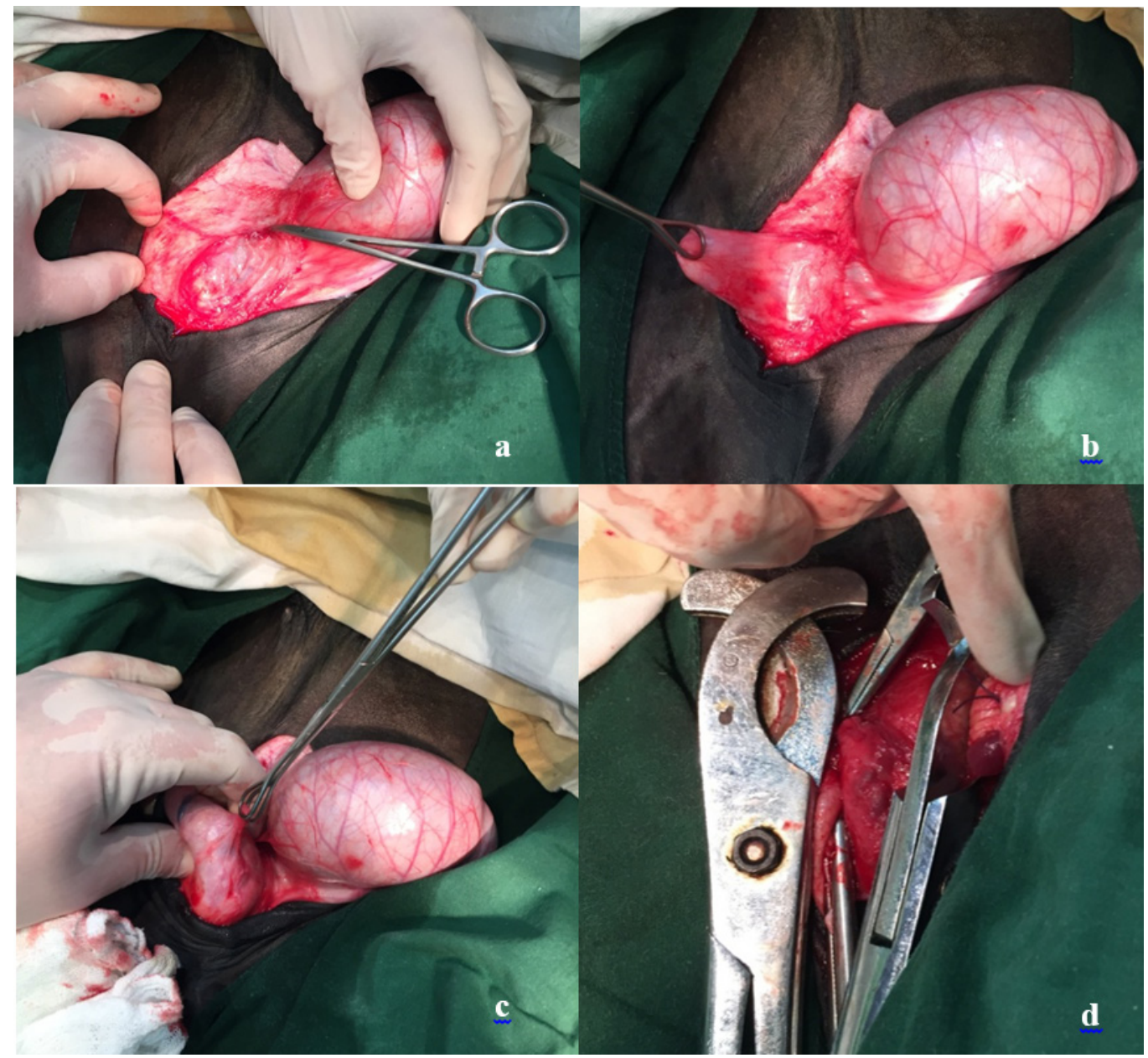

Fig. 3. - Cryptorchidectomy via inguinal approach (a- superficial inguinal ring; b - retracting of gubernaculum testis exposes the vaginal process; c -testis retracted extra-abdominal; $d$-spermatic cord hemostasis.

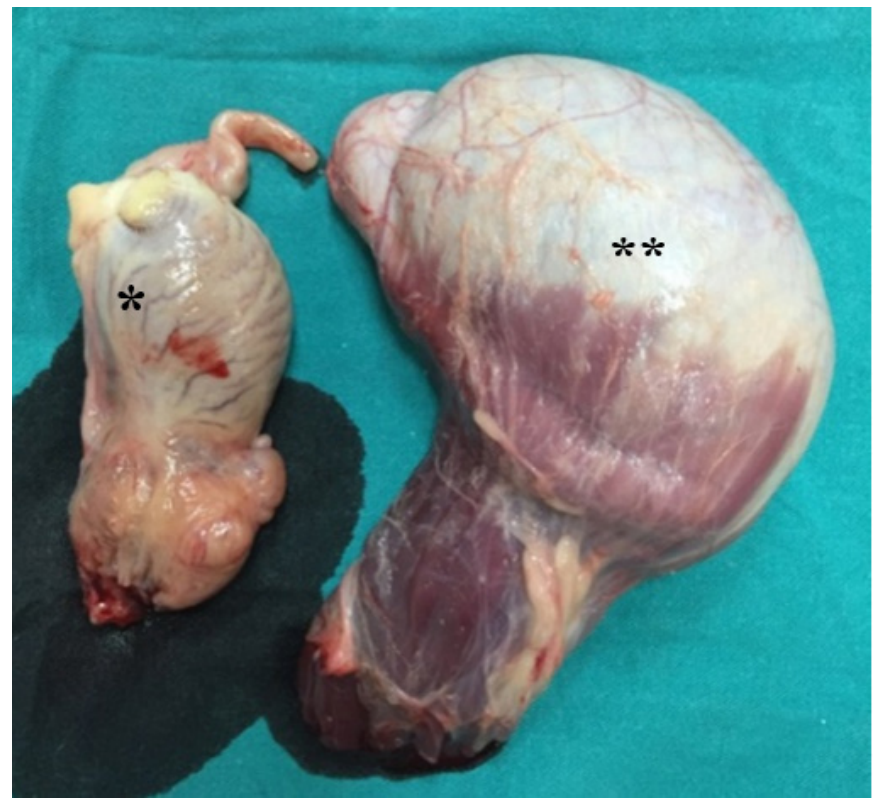

Fig. 4. - Testis after removal ${ }^{*}$ - right hypoplastic testis; ${ }^{* *}$ - left normal testis) 
cannot be recommended as a trustworthy method for castration of inguinal cryptorchid stallions (Bergeron et al., 1998; Voermans et al., 2006).

In Cribb et al. (2015) opinion, horses undergoing laparoscopic cryptorchidectomy had increased surgical preparation time, increased surgery and anesthesia times, and more postoperative complications, compared with horses undergoing open cryptorchictomy, therefore laparoscopy may be advantageous for a second attempt at cryptorchidectomy or if the testicle location is unknown prior to surgery.

Using a noninvasive inguinal approach is a consecrated method and widely accepted for inguinal cryptorchidectomy (Adams and Fessler, 2000; Arighi et al., 1988; Moore et al., 1978; Rodgerson and Hanson, 1997; Turner and McIlwraith, 1989; Valdez et al., 1979; Walker and Vaughan, 1980).

Method of eversion into the inguinal canal of the inverted vaginal process into the abdomen, applied in this case report, has been described in the literature (Arighi et al., 1988; Moore et al., 1978; Rodgerson and Hanson, 1997; Turner and McIlwraith, 1989; Valdez et al., 1979). As previously mentioned, according to our knowledge, this report describes the performance of this new technique on stallions for the first time in Romania.

\section{CONCLUSIONS}

If the location of the testis is unknown, diagnostic laparoscopy in standing position can be decisive.

Cryptorchidectomy via inguinal approach and noninvasive method for identifying and everting the vaginal process by traction of the inguinal extension of the gubernaculum testis are commonly facile procedures.

Our observations can be included amongst the few reports which states that standing laparoscopic criptorchidectomy can not be performed in stallions, including miniature stallions.

Acknowledgements. This research work was carried out with the support of the project „Dezvoltarea infrastructurii de cercetare, educaţie si servicii in domeniile medicinei veterinare si tehnologiilor inovative pentru RO 05", cod SMIS-CSNR 2669

\section{REFERENCES}

1. Adams S, Fessler F (2000). Atlas of equine surgery, Ed. W.B. Saunders Comp., Philadelphia

2. Arighi M, Horney JD, Bosu WT (1988). Noninvasive Inguinal Approach for Cryptorchidectomy in Thirty-eight Stallions. Can Vet 29(4), 346-9.

3. Bergeron JA, Hendrickson DA, McCue PM (1998). Viability of an inguinal testis after laparoscopic cauterization and transection of its blood supply. J Am Vet Med Assoc 213(9), 1303-1304, 1280.

4. Cox JE, Neal PA, Edwards GB (1978). Suprapubic paramedian laparotomy for equine abdominal cryptorchidism. J Am Vet Med Assoc 173(6), 680-682.

5. Cribb NC, Koenig J, Sorge U (2015). Comparison of laparoscopic versus conventional open cryptorchidectomies on intraoperative and postoperative complications and duration of surgery, anesthesia, and hospital stay in horses. J Am Vet Med Assoc 246(8), 885892. doi: 10.2460/javma.246.8.885.

6. Davis EW (1997). Laparoscopic cryptorchidectomy in standing horses. Vet Surgery 26(4), 326-31.

7. Fischer AT Jr, Vachon AM (1992). Laparascopic cryptochidectomy in horses. J Am Vet Med Assoc 201(11), 1705-1708.

8. Fischer AT Jr. (1991). Standing laparascopic surgery. Vet Clin North Am Equine Pract7, 641-647

9. Gardner AK, Santschi EM, Aeffner F, Pigott JH, Russell DS (2016). Testicular ischaemic necrosis as a cause of equine cryptorchidism. Equine Veterinary Education doi: $10.1111 /$ eve. 12544

10. Hanrath M, Rodgerson DH (2002). Laparoscopic Cryptorchidectomy Using Electrosurgical Instrumentation in Standing Horses, Vet. Surgery 31:117-124. doi:10.1053/ jvet.31049.

11. Hendrickson DA, Wilson DG (1997). Laparascopic cryptorchid castration in standing horses. Vet. Surgery 26(4), 335-339.

12. Hendrickson DA (2012). A review of equine laparoscopy. ISRN Vet Science doi: 10.5402/2012/492650

13. Hendrickson DA (2006). Laparascopic cryptochidectomy and ovariectomy in horses. Vet Clin North Am 22(3), 777798.

14. McKinnon OA, Squires LE, Vaala EW, Varner DD (2011). Equine reproduction, 2th ed. Ed Wiley-Blackwell

15. Moore JN, Johnson JH, Tritschler LG, Garner HE (1978). Equine Cryptorchidism: Pre-surgical Considerations and Surgical Management. Veterinary Surgery 7, 43-47. doi: 10.1111/j.1532-950X.1978.tb00578.x

16. Muir WW 3rd, Lerche P, Robertson JT, Hubbell JA, Beard W, Miller T, Badgley B, Bothwell V (2000). Comparison of four drug combinations for total intravenous anesthesia of horses undergoing surgical removal of an abdominal testis. J Am Vet Med Assoc 217(6), 869-873.

17. Palmer SE, Passmore JL (1987). Midline scrotal ablation technique for unilateral cryptorchid castration in horses. J Am Vet Med Assoc 190(3), 283-285. 
18. Ragle CA, Southwood LL, Howlett MR (1998). Ventral abdominal approach for laparoscopic cryptochidectomy in horses. Vet Surg 27(2), 138-142.

19. Rodgerson HD, Hanson R (1997). Criptorchidism in Horses. Part 2. Treatment. Cont Educ 19(12), 1372-1395

20. Searle D, Dart AJ, Dart CM, Hodgson DR (1999). Equine castration: review of anatomy, approaches, techniques and complications in normal, cryptorchid and monorchid horses. Aust Vet J 77(7), 428-34.

21. Shira MJ, Genetzky RM (1982). Equine Chryptorchidism, Iowa State University Veterinarian 44(2), 77-81.

22. Swift PN (1972). Castration of a stallion with bilateral abdominal cryptorchidism by flank laparotomy. Aust Vet J 48(8), 472-473.

23. Turner AS, McIlwraith CW (1989). Tecniques in Large Animal Surgery ed. 2, Ed Lea\&Febiger, Philadelphia 185191.

24. Valdez H, Taylor TS, McLaughlin SA, Martin MT (1979) Abdominal cryptochidectomy in the horse, using inguinal extension of the gubernaculum testis. J Am Vet Med Assoc 174, 1110-1112.

25. Van der Velden MA (1990). Cryptorchism in the horse. Tijdschr Diergeneeskd 115(9), 399-409.

26. Voermans M, Rijkenhuizen AB, van der Velden MA (2006). The complex blood supply to the equine testis as a cause of failure in laparoscopic castration. Equine Vet J 38(1), 35-9.

27. Walker DF, Vaughan JT (1980). Bovine and Equine Urogenital Surgery. Ed. Lea \& Febiger, Philadelphia

28. Wilson DG, Hendrickson DA, Cooley AJ, Degrave-Madigan E (1996). Laparascopic methods for castration of equids. J Am Vet Med Assoc 209 (1), 112-114.

29. Wilson DG, Madison WI (1989). Laparascopy as an aid in the surgical management of the equine hemicastrate. Am Assoc of Equine Pract 35, 347-353.

30. Wilson DG, Reinertson EL (1987). A modified parainguinal approach for cryptorchidectomy in horses. An evaluation in 107 horses. Vet Surgery 16(1), 1-4. 